\title{
Portfolio optimization by improved NSGA-II and SPEA 2 based on different risk measures
}

\author{
Massimiliano Kaucic ${ }^{*}$ (D), Mojtaba Moradi ${ }^{2}$ and Mohmmad Mirzazadeh ${ }^{3}$
}

\author{
${ }^{*}$ Correspondence: \\ massimiliano.kaucic@deams.units.it \\ 'Department of Economics, \\ Business, Mathematics and \\ Statistics, University of Trieste, \\ Piazzale Europa 1, 34127 Trieste, \\ Italy \\ Full list of author information is \\ available at the end of the article
}

\begin{abstract}
In this study, we analyze three portfolio selection strategies for loss-averse investors: semi-variance, conditional value-at-risk, and a combination of both risk measures. Moreover, we propose a novel version of the non-dominated sorting genetic algorithm Il and of the strength Pareto evolutionary algorithm 2 to tackle this optimization problem. The effectiveness of these algorithms is compared with two alternatives from the literature from five publicly available datasets. The computational results indicate that the proposed algorithms in this study outperform the others for all the examined performance metrics. Moreover, they are able to approximate the Pareto front even in cases in which all the other approaches fail.
\end{abstract}

Keywords: Multi-objective portfolio optimization, Semi-variance, CVaR, NSGA-II, SPEA 2, Intermediate crossover, Gaussian mutation

\section{Introduction}

The portfolio selection problem can be defined as the optimal allocation of wealth among a finite number of assets that follows careful processing of all available information about both investors and markets (Meucci 2009). Markowitz's mean-variance model is by far the most popular procedure in asset allocation (Guerard 2009). Even if it is considered the cornerstone in this field, the mean-variance portfolio optimization model presents two serious drawbacks from a theoretical point of view. First, when asset returns are skewed and fat-tailed, they tend to include only a limited proportion of stochastically dominant assets in the efficient solutions, and prematurely preclude asset with negatively skewed returns. Second, risk is measured by variance, which treats both the above and below target returns equally, while investors are more concerned about the probability of investment returns falling below the target return. Consequently, risks are under-estimated and portfolios that are downside efficient are ruled out.

A solution proposed in the literature to make Markowitz's approach more effective is to replace the variance with a downside risk measure in order to model the loss aversion of investors properly. In this context, semi-variance has been studied extensively (Nawrocki 1999; Sing and Ong 2000). Two main reasons justify these efforts. First, semivariance is an approximation of the skewness for the return distribution, since it measures below-target returns. The higher is the value of semi-variance, the greater are both the

(c) The Author(s). 2019 Open Access This article is distributed under the terms of the Creative Commons Attribution 4.0 International License (http://creativecommons.org/licenses/by/4.0/), which permits unrestricted use, distribution, and reproduction in any medium, provided you give appropriate credit to the original author(s) and the source, provide a link to the Creative Commons license, and indicate if changes were made. 
degree of negative skewness and the risk of the investment. Second, semi-variance efficient portfolios closely approximate the stochastic-dominance efficient set. However, the computation of portfolio semi-variance is a difficult task owing to the endogenous nature of the portfolio co-semi-variance matrix, which depends on the weights assigned to each asset, that is, changes in the weights affect the periods in which the portfolio underperforms the target level, which, in turn, affects the evaluation of the co-semi-variance matrix itself. Some attempts have been proposed to solve this problem directly (Hogan and Warren 1972; Konno et al. 2002; Markowitz 1959; Markowitz et al. 1993). Other studies have focused on the definition of an exogenous co-semi-variance matrix that satisfactorily approaches the endogenous one (Ballestero and Pla-Santamaria 2005; Cumova and Nawrocki 2011; Estrada 2008; Nawrocki 1991). Nowadays, because of the regulatory importance of quantifying large losses in banking and insurance, another class of downside risk measures, called quantile-based measures, occupies a leading position in the risk management sector. One of the most popular examples is value-at-risk (VaR), defined as the maximum loss occurring over a given period at a given confidence level. Although $\mathrm{VaR}$ is apparently easy to use and intuitive, it presents several disadvantages. Primarily, it ignores losses exceeding $\mathrm{VaR}$ and is not sub-additive, that is, diversification of the portfolio may increase VaR (Artzner et al. 1999). From a computational perspective, VaR is difficult to use when investors want to optimize their portfolios, since it is represented by a non-linear, non-convex, and non-differentiable function with multiple local optima (Gaivoronski and Pflug 2005). Moreover, rational agents wishing to act on their decisions according to expected utility theory may be misled by the information related to the portfolio VaR (Yamai et al. 2002). To deal with these shortcomings, (Rockafellar et al. 2000) introduced the conditional value-at-risk (CVaR), which is defined as the conditional expectation of losses above the VaR (Sarykalin et al. 2008). CVaR is a coherent risk measure in the sense of (Artzner et al. 1999) and, because it is a convex function, optimization problems with CVaR as the minimization objective and/or constraints can be efficiently handled (Krokhmal et al. 2002; Larsen et al. 2002; Rockafellar and Uryasev 2002).

Increasing complexity of practical applications has led researchers to develop heuristic procedures for solving their portfolio optimization problems. These techniques require less domain information to be considered than the standard gradient-based mathematical programming methods do. Moreover, they guarantee satisfactory approximations to solutions in a fair computational time even when they deal with non-convexity, discontinuity, and integer decision variables. The approaches that have been proposed in the soft-computing literature can be categorized into the following two groups. On one hand, single objective methods optimize a weighted sum of the portfolio objectives. On the other hand, multi-objective evolutionary algorithms (MOEAs) attempt to tackle the allocation problem directly in its multi-objective form by simultaneously optimizing risk and reward. In the first case, the complete set of risk-return profiles is obtained by varying a parameter that represents the risk aversion of the investor (Chang et al. 2000; Crama and Schyns 2003; Cura 2009; Woodside-Oriakhi et al. 2011). In the second case, the complete efficient frontier is represented in a single run (Anagnostopoulos and Mamanis 2011a; Meghwani and Thakur 2017; Mishra et al. 2014). Both categories pay great attention to encoding types and constraint-handling techniques (Liagkouras and Metaxiotis 2015; Meghwani and Thakur 2017; Metaxiotis and Liagkouras 2012; Ponsich et al. 2013). Some real-life situations, which are not considered in Markowitz's model, have 
been analyzed recently by (Eftekharian et al. 2017) and (Meghwani and Thakur 2018). (Eftekharian et al. 2017) include as constraints some restrictions on the number of assets in the portfolio, limitations on investing in assets from a given industry, and cardinality, class, and quantity constraints. Furthermore, they developed an improved version of the NSGA II algorithm, called 2-Phase NSGA II, to solve the resulting optimization problem. Meghwani and Thakur (2018) focus on the problem of handling equality constraints, like self-financing constraints, and constraints arising from the inclusion of transaction cost models using MOEAs. Researchers have also focused on so-called swarm intelligence methods to overcome the computational difficulties of realistic portfolio designs. Unlike evolutionary algorithms that utilize the principle of natural selection, these approaches are inspired by the behavior and self-organizing interaction among agents, such as foraging of ant and bee colonies, bird flocking, and fish schooling (see Ertenlice and Kalayci (2018) for a detailed review of the subject).

Over the past few decades, machine-learning algorithms have been widely used to explore financial data and make data-driven predictions (Chao et al. 2019; Kou 2019). For instance, (Huang and Kou 2014) present a kernel entropy manifold learning algorithm to measure the relationships between two financial data points in order to describe the characteristics of a financial system by deriving the dynamic properties of the original data space. Similarly, (Huang et al. 2017) propose an information metric-based manifold learning algorithm to extract the intrinsic manifold of a dynamic financial system and to detect impending crises. (Ergu et al. 2014) focus on the use of the analytical network process in risk assessment and decision analysis of an emergent event. They propose a new consistency index to assess the key factors of risks and analyze the impacts and preferences of decision alternatives. Zhang et al. (2019) develop three soft minimum cost models based on different weighted average operators for resolving consensus decision-making problems. The usefulness of the proposed models is validated in a real-world loan consensus problem, using data from a Chinese P2P platform.

Kalayci et al. (2019) review state-of-the-art methods dedicated to mean-variance portfolio optimization.

Evolutionary computations are made by iterations and, in each iteration, the weights of the portfolios are known a priori because they are generated by the algorithm. Thus, it is possible to directly determine in which periods the portfolio underperforms the target level. In this manner, the difficulty of computing the ex-post portfolio semi-variance can be fruitfully resolved by the use of an evolutionary algorithm. One of the first studies to attempt this is (Dueck and Winker 1992), who reformulate the bi-objective optimization problem into a single-objective and solve it through a local search technique, called threshold accepting. In a similar way, (Arnone et al. 1993) propose a solution to a parametric programming problem with the objective of optimizing a convex combination of mean and semi-variance. The algorithm is based on Whitley's GENITOR system (Whitley 1988) and uses a steady-state breeding strategy and elitist selection. Chang et al. (2009) apply a genetic algorithm to portfolio optimization problems in different risk measures, namely variance, semi-variance, absolute deviation, and variance with skewness. Recently, (Liagkouras and Metaxiotis 2013) address the mean-semi-variance portfolio optimization problem from a multi-objective perspective by developing an ad-hoc evolutionary algorithm. Evidence of the robustness of the algorithm is accomplished in out-of-sample testing during both bull and bear market conditions on the FTSE 100. 
Macedo et al. (2017) compare the non-dominated sorting genetic algorithm II (NSGAII, Deb et al. (2002)) and the strength Pareto evolutionary Algorithm 2 (SPEA 2, Zitzler et al. (2001)) within the mean-semi-variance portfolio optimization framework. Numerical experiments indicate that NSGA-II outperforms SPEA 2 in-sample. Senhaji et al. (2016) propose to resolve the problem by combining the continuous Hopfield neural network with NSGA-II. The effectiveness of this strategy is proved using a portfolio of 20 assets.

Regarding the quantile-based minimization problems involving many financial instruments, a large number of price scenarios is necessary to estimate risk correctly. As the dimension of the problems increases, this operation can be time consuming (Lim et al. 2010) and the use of heuristics may be advisable or even necessary to detect a solution. An example is provided by the multi-purpose data-driven optimization heuristic proposed in Gilli M and Hysi (2006), which deals with different risk functions, such as VaR, CVaR, maximum loss, and Omega, as well as with practical constraints on the portfolio composition. An evolutionary computation approach is developed by (Hochreiter 2007) to solve the general scenario-based risk-return portfolio optimization problem when standard deviation, VaR, or CVaR are used to represent the risk of the investment. A variant of the NSGA-II algorithm based on differential operators is developed by (Krink and Paterlini 2011) for portfolio optimization involving real-world constraints and quantilebased risk measures. Meanwhile, a comparison of the capabilities of different MOEAs to adapt in any addition of new constraints and/or replacement of the risk function is given in (Anagnostopoulos and Mamanis 2011b), and (Baixauli-Soler et al. 2012) focus on SPEA 2 performance. Recently, hybrid stock trading systems based on evolutionary metaheuristics and mean-CVaR models are proposed in Chen and Wang (2015) and (Qin et al. 2014).

The contribution of this study is twofold. On one hand, based on the promising results of (Subbu et al. 2005) and (Baixauli-Soler et al. 2010), we include in the same portfolio optimization framework the loss-averse attitude of investors as well as the capital requirements imposed by the regulator, and we investigate the relationship between semivariance and CVaR in quantifying the downside risk. On the other hand, we extend the research of (Liu et al. 2010) and investigate the effectiveness of using the uniform selection scheme, the extended intermediate crossover operator (Gen and Cheng 2000; Mühlenbein and Schlierkamp-Voosen 1993), and the Gaussian mutation (Hinterding 1995; Schwefel 1987) in the NSGA-II and SPEA 2 algorithms in order to generate the approximated Pareto fronts for the considered downside risk-based portfolio optimization problems. In addition, the performance of the proposed algorithms is compared to that of other variants of the NSGA-II and SPEA 2 algorithms that have already been shown to be highly competitive in portfolio optimization problems. The results on five publicly available datasets show that our procedures completely dominate the fronts produced by the counterparts from the literature. Furthermore, our variants of the NSGA-II and SPEA 2 algorithms can generate the entire Pareto front for large-scale problems for which the other Pareto-based approaches are unable to work properly.

The rest of the paper is organized as follows. In "The portfolio selection problem under downside risk measures" section, we introduce three preference relations based on the reward-downside risk principle and discuss the related portfolio selection problems. "Pareto-based evolutionary algorithms" section presents a description of the MOEAs 
used for solving the resulting optimization problems. "Experimental analysis" section outlines the numerical experiments and "Concluding remarks and future research" section presents concluding remarks and ideas for further research.

\section{The portfolio selection problem under downside risk measures}

Consider a financial market consisting of $\mathrm{n}$ risky assets, indexed from 1 to $\mathrm{n}$, and modeled by a probability space $(\Omega, \mathfrak{F}, P)$. Let agents act their investment decisions over a one-period horizon with respect to the following set of feasible, or admissible, portfolios defined according to the budget constraint and no-short selling:

$$
\mathcal{X}=\left\{\left(x_{1}, \ldots, x_{n}\right) \in \mathbb{R}^{n}: \sum_{i=1}^{n} x_{i}=1, x_{i} \geq 0, i=1, \ldots, n\right\}
$$

where $x_{i}$ represents the proportion of capital to be allocated to asset $i$, with $i=1, \ldots, n$.

The random rate of return of asset $i$ at the end of the investment period, denoted as $R_{i}$, is assumed to have a continuous probability density function (pdf). Hence, the rate of return of a portfolio $\mathbf{x}=\left(x_{1}, \ldots, x_{n}\right)^{T} \in \mathcal{X}$ is the random variable $R(\mathbf{x})=\sum_{i=1}^{n} R_{i} x_{i}$ with $\operatorname{pdf} f_{R(\mathbf{x})}$ induced by that of $\left(R_{1}, \ldots, R_{n}\right) . R(\mathbf{x})$ is measurable in $\left(R_{1}, \ldots, R_{n}\right)$ with expected rate of return defined as

$$
\mathbb{E}(R(\mathbf{x}))=\sum_{i=1}^{n} \mathbb{E}\left(R_{i}\right) x_{i}
$$

where $\mathbb{E}(\cdot)$ denotes the expectation and $\mathbb{E}\left(R_{i}\right)$ is the expected rate of return of asset $i$. Given a level $z$ for the rate of return, the cumulative distribution function (cdf) of $R(\mathbf{x})$ is defined as $F_{R(\mathbf{x})}(z)=P(R(\mathbf{x}) \leq z)=\int_{\{R(\mathbf{x}) \leq z\}} f_{R(\mathbf{x})}(r) d r$. It is also assumed that $F_{R(\mathbf{x})}$ is continuous and strictly increasing with respect to $z \in \mathbb{R}^{1}$. The portfolio loss distribution is defined as the negative of the portfolio return distribution, that is, $L(\mathbf{x})=-R(\mathbf{x})$.

To identify the portfolio in $\mathcal{X}$ that guarantees the "best" rate of return, a model for preferences under uncertainty needs to be defined. We adopt the so-called reward-risk approach that relates the portfolio selection problem to a multi-objective optimization problem in two steps. First, a set of objectives that the investor perceives as beneficial is identified, and second, a set of objectives he or she considers damaging is identified in relation to $R(\cdot)$. Then, a preference relation is defined based on these criteria as follows.

Definition 1 Let $f_{1}, \ldots, f_{p}: \mathcal{X} \rightarrow \mathbb{R}$ and $g_{1}, \ldots, g_{q}: \mathcal{X} \rightarrow \mathbb{R}$ be the reward-type and the risk-type objective functions, respectively. Then for all $\mathbf{x}, \mathbf{y} \in \mathcal{X}$, we say that $R(\mathbf{x})$ dominates (is preferred to) $R(\mathbf{y})$ if and only if $f_{i}(\mathbf{x}) \geq f_{i}(\mathbf{y})$ for all $i=1, \ldots, p$ and $g_{j}(\mathbf{x}) \leq g_{j}(\mathbf{y})$ for all $j=1, \ldots, q$ with at least one strict inequality. Alternatively, we can say that portfolio $\mathbf{x}$ dominates portfolio $\mathbf{y}$.

According to this definition, a portfolio $\mathbf{x}^{*} \in \mathcal{X}$ is (Pareto) optimal if and only if it is non-dominated with respect to $\mathcal{X}$, that is, another $\overline{\mathbf{x}} \in \mathcal{X}$ that dominates $\mathbf{x}^{*}$ does not exist. Thus, an efficient portfolio in the reward-risk model is a Pareto optimal solution of the following multi-objective problem:

$$
\begin{array}{ll}
\text { minimize } & F(\mathbf{x})=\left(-f_{1}(\mathbf{x}), \ldots,-f_{p}(\mathbf{x}), g_{1}(\mathbf{x}), \ldots, g_{q}(\mathbf{x})\right) \\
\text { subject to } & \mathbf{x} \in \mathcal{X} .
\end{array}
$$


The solutions to Problem (3) form the so-called efficient set, or Pareto optimal set, of which image in the reward-risk space is called efficient frontier, or Pareto front. Under certain smoothness assumptions, it can be induced from the Karush-Kuhn-Tucker conditions that, when the $p+q$ objectives are continuous, the efficient frontier defines a piecewise continuous $(p+q-1)$-dimensional manifold in the decision space (Li and Zhang 2009). Therefore, the efficient frontier of a continuous bi-objective portfolio optimization problem is a piecewise continuous 1-D curve in $\mathbb{R}^{2}$, while, in the case of a problem with three objectives, it is a piecewise continuous 2-D surface in $\mathbb{R}^{3}$.

We now specialize this general reward-risk framework by considering investors that focus on the mean rate of return (2) as the reward criterion and employ different measures to assess the downside risk of their investments.

\section{The mean-semi-variance model}

One of the most well-known downside risk measures of a portfolio $\mathbf{x}$ is its semi-variance, already introduced in the seminal paper of (Markowitz 1959) and defined as

$$
V^{-}(R(\mathbf{x}))=\mathbb{E}\left(\left([R(\mathbf{x})-b]^{-}\right)^{2}\right)
$$

where $[u]^{-}=\max \{0,-u\}$ and $b$ represents a given benchmark, for example, the portfolio expected rate of return, the Roy's safety first criterion, or a Treasury rate of return. In our analysis, we set the target level $b$ equal to 0 in order to estimate the variance of portfolio losses. As proved in Fishburn (1977), this downside risk measure is consistent with utility theory

An investor that operates her or his decisions according to the mean-semi-variance model is interested in maximizing the expected rate of return of her or his portfolio and, simultaneously, minimizing its semi-variance:

$$
\begin{array}{ll}
\text { minimize } & \left(-\mathbb{E}(R(\mathbf{x})), V^{-}(R(\mathbf{x}))\right) \\
\text { subject to } & \mathbf{x} \in \mathcal{X} .
\end{array}
$$

\section{The mean-CVaR model}

The CVaR of a portfolio $\mathbf{x}$ at the confidence level $\alpha$, with $\alpha \in(0,1)$, represents the average of losses in the $100(1-\alpha) \%$ worst scenarios (Acerbi and Tasche 2002). According to our assumptions about the cdf of $R(\mathbf{x})$ and the definition of the portfolio loss $L(\mathbf{x})$ as the opposite of $R(\mathbf{x})$, this downside risk measure is defined as

$$
C \operatorname{VaR}_{\alpha}(L(\mathbf{x}))=\mathbb{E}\left(L(\mathbf{x}) \mid L(\mathbf{x}) \geq \operatorname{VaR}_{\alpha}(L(\mathbf{x}))\right),
$$

where $\operatorname{VaR}_{\alpha}(L(\mathbf{x}))=\sup \{z \mid P(L(\mathbf{x}) \geq z) \leq \alpha\}=F_{L(\mathbf{x})}^{-1}(1-\alpha)$ is the value-at-risk at the confidence level $\alpha$ of the portfolio $\mathbf{x}$.

An agent whose preferences are dictated by the mean-CVaR model would select her or his portfolio weights according to the following bi-objective optimization problem:

$$
\begin{array}{ll}
\operatorname{minimize} & \left(-\mathbb{E}(R(\mathbf{x})), \operatorname{CVaR}_{\alpha}(L(\mathbf{x}))\right) \\
\text { subject to } & \mathbf{x} \in \mathcal{X} .
\end{array}
$$

\section{The mean-semi-variance-CVaR model}

Using only one criterion to model risk in a portfolio selection model may provide a restricted picture of the assessment process (Steuer et al. 2005). It is advisable to include 
multiple risk measures in order to cover the different facets of an investor's preferences. In this study, we analyze the combined effects of CVaR and semi-variance. The related tri-objective optimization problem is

minimize $\left(-\mathbb{E}(R(\mathbf{x})), V^{-}(R(\mathbf{x})), C \operatorname{VaR}_{\alpha}(L(\mathbf{x}))\right)$

subject to $\mathbf{x} \in \mathcal{X}$,

where $V^{-}$and $C V a R_{\alpha}$ are the same as the definitions in (4) and (6), respectively.

In this manner, the preferences are modeled as follows.

Definition 2 Portfolio $\mathbf{x}$ is preferred to portfolio $\mathbf{y}$ if and only if $\mathbb{E}(R(\mathbf{x})) \geq$ $\mathbb{E}(R(\mathbf{y})), C V a R_{\alpha}(L(\mathbf{x})) \leq C \operatorname{VaR}_{\alpha}(L(\mathbf{y}))$ and $V^{-}(R(\mathbf{x})) \leq V^{-}(R(\mathbf{y}))$, with at least one strict inequality.

This model may produce improved solutions when a mean-CVaR efficient portfolio has an excessively large semi-variance or when a mean-semi-variance efficient portfolio has an excessively large CVaR.

\section{Scenario-based framework for portfolio optimization}

A practical way to handle the random variables that represent assets and portfolio rates of return is to treat them as discrete. Therefore, let $\Omega=\left\{\omega_{1}, \ldots, \omega_{S}\right\}, P\left(\omega_{s}\right)=p_{s}$, with $s=$ $1, \ldots, S$ and $\sum_{s=1}^{S} p_{s}=1, \mathcal{F}$ be a given $\sigma$-field and assume the following table of scenarios is available

$$
\left(\begin{array}{lll}
\mathbf{r}_{1} & \ldots & \mathbf{r}_{S} \\
p_{1} & \ldots & p_{S}
\end{array}\right)
$$

where $S$ represents the number of involved scenarios, $\mathbf{r}_{s}=\left(r_{1 s}, \ldots, r_{n s}\right)^{T}$ is the $n$-vector of rates of return for the $s$-th scenario, and $p_{s}$ is the associated probability of occurrence, with $s=1, \ldots, S$. Assume that $p_{s}=1 / S$ for all $s$. Thus, the rate of return of $\mathbf{x} \in \mathcal{X}$ may assume $S$ values calculated as $r_{s}^{p}(\mathbf{x})=\sum_{i=1}^{n} r_{i s} x_{i}$. Analogously, assuming that the distribution of the portfolio rate of return $R(\mathbf{x})$ is such that $P\left(R(\mathbf{x})=r_{s}^{p}(\mathbf{x})\right)=1 / S$ with $s=1, \ldots, S$, since the expected rate of return for the $i$-th security can be computed as the mean rate of return over the $S$ scenarios (i.e., $\mathbb{E}\left(R_{i}\right)=\frac{1}{S} \sum_{s=1}^{S} r_{i s}$ ), the expected rate of return of portfolio $\mathbf{x}$ becomes

$$
\mathbb{E}(R(\mathbf{x}))=\sum_{i=1}^{n} \mathbb{E}\left(r_{i}\right) x_{i}=\frac{1}{S} \sum_{s=1}^{S} r_{s}^{p}(\mathbf{x}) .
$$

Now it is possible to reformulate the previously introduced downside risk-based portfolio allocation models in terms of the scenarios conveyed in (9).

Based on the findings of (Cumova and Nawrocki 2011), we estimate $V^{-}(R(\mathbf{x}))$ by means of the so-called co-semi-variance matrix, henceforth denoted as $C^{-}=\left(C_{i j}^{-}\right)$, which is defined element-wise as 


$$
\begin{aligned}
& C_{i j}^{-}=\frac{1}{S} \sum_{s=1}^{S}\left(r_{i s}-b\right) \min \left\{r_{j s}-b, 0\right\} \\
& C_{j i}^{-}=\frac{1}{S} \sum_{s=1}^{S}\left(r_{j s}-b\right) \min \left\{r_{i s}-b, 0\right\}
\end{aligned}
$$

for all $i, j=1, \ldots, n$. It can be noted that $C^{-}$is an asymmetric matrix, since in general, assets $i$ and $j$ do not have the same below-target returns during the same period, that is, $C_{i j}^{-} \neq C_{j i}^{-}$. The semi-variance of portfolio $\mathbf{x}$ can then be calculated as

$$
V^{-}(R(\mathbf{x}))=\sum_{i=1}^{n} \sum_{j=1}^{n} C_{i j}^{-} x_{i} x_{j} .
$$

In this manner, Problem (5) translates into the maximization of a linear objective function, given by Eq. (10), and the simultaneous minimization of a quadratic objective function, given by Eq. (13), over the unit standard $n$-dimensional simplex. Regarding Problem (7), the CVaR expression (6) can be evaluated as an arithmetic mean over scenarios as follows. Let $l_{s}^{p}(\mathbf{x})=-r_{s}^{p}(\mathbf{x})$ be the portfolio loss in the $s$-th scenario, after sorting losses in ascending order, that is, $l_{(1)}^{p}(\mathbf{x}) \leq l_{(2)}^{p}(\mathbf{x}) \leq \ldots \leq l_{(S)}^{p}(\mathbf{x})$, the $\operatorname{VaR}_{\alpha}$ can then be defined as $l_{(\lfloor\alpha\rfloor\rfloor)}^{p}(\mathbf{x})$ and $C V a R_{\alpha}$ can be estimated as

$$
\begin{aligned}
\operatorname{CVaR}_{\alpha}(L(\mathbf{x})) & =\frac{\sum_{s=1}^{S} l_{(s)}^{p}(\mathbf{x}) \mathbb{I}\left(r_{(s)}^{p}(\mathbf{x}) \geq \operatorname{VaR}_{\alpha}(\mathbf{x})\right)}{\sum_{s=1}^{S} \mathbb{I}\left(l_{(s)}^{p}(\mathbf{x}) \geq \operatorname{VaR}_{\alpha}(\mathbf{x})\right)} \\
& =\frac{1}{(1-\alpha) S}\left[\sum_{s=\lceil\alpha S\rceil+1}^{S} l_{(s)}^{p}(\mathbf{x})+(\lceil\alpha S\rceil-\alpha S) l_{(\lceil\alpha S\rceil)}^{p}(\mathbf{x})\right]
\end{aligned}
$$

where $\mathbb{I}(\cdot)$ represents the indicator function. In this case, the investor maximizes the expected rate of return as given in Eq. (10) and minimizes the risk calculated according to Eq. (14).

Finally, Problem (8) simultaneously exploits Eqs. (10), (13), and (14) in the optimization process.

\section{Pareto-based evolutionary algorithms}

The capabilities of MOEAs to generate reasonably good approximations of the Pareto front in a single run and within a limited computational time have already been shown in the literature on the mean-variance portfolio selection problem.

In this study, we focus on two variants of popular algorithms, NSGA-II and SPEA 2, and investigate their effectiveness in solving the portfolio optimization problems formulated in the previous section. These algorithms belong to the family of Pareto-based MOEAs and include a two-level ranking scheme to guide the search toward the true Pareto front (Emmerich and Deutz 2018). The first ranking is provided by the Pareto dominance relation, while the second concerns the diversity of the solutions and applies to the individuals that share the same position in the first ranking. However, the methods by which NSGAII and SPEA 2 approximate the true Pareto front differ and the corresponding procedures are described in the following overview ${ }^{2}$. 
NSGA-II

The NSGA-II procedure is described in Algorithm 1. The crowding distance mechanism is employed to preserve the diversity of solutions. It evaluates the volume of the hyper-rectangle defined by two nearest neighbors (Zitzler and Thiele 1999). New solutions, called offsprings, are generated using a selection mechanism and a set of variation operators. Based on the values provided by the ranking scheme, the best individuals from the combination of the current population $P_{k}$ and the offspring pool $Q_{k}$ are detected and those with lower rank and higher crowding distance are saved in the next population $P_{k+1}$. In case some candidate solutions are of the same rank and not all of them enter the next population, the less crowded individuals from that particular rank are selected to fit the next population, thereby ensuring elitism.

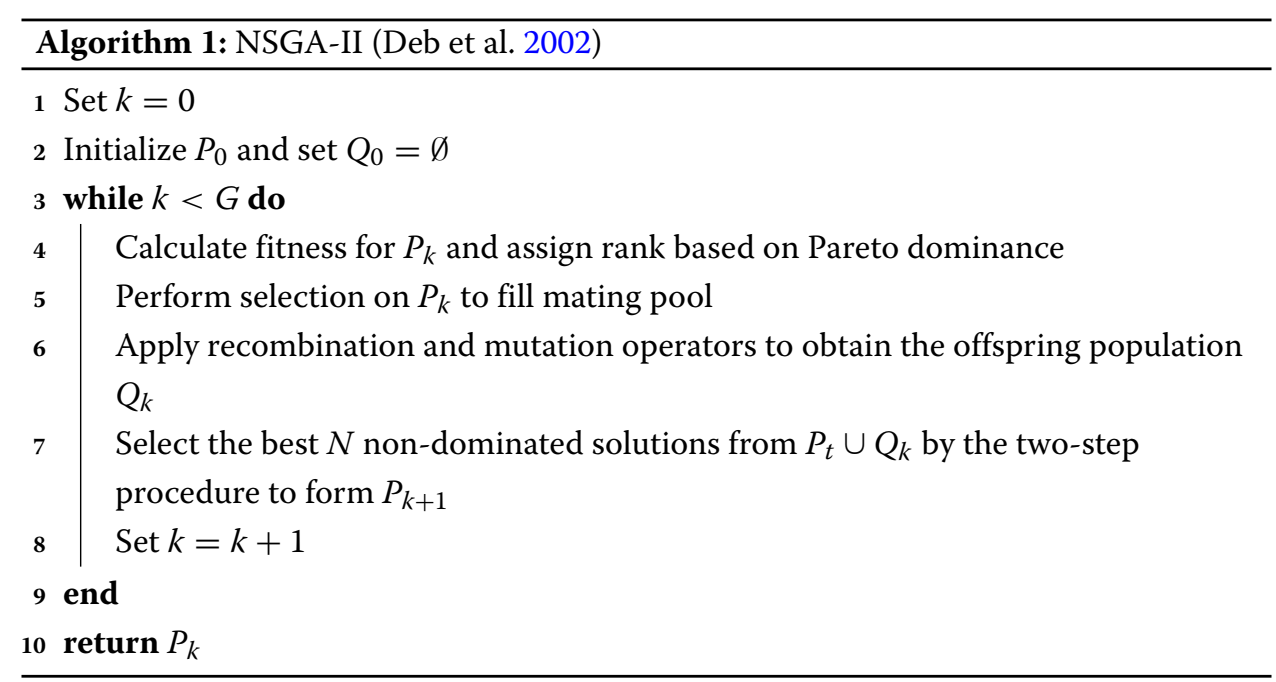

\section{SPEA 2}

As reported in Algorithm 2, SPEA 2 first initializes a population of candidate solutions $P_{k}$, then stores the best solutions in an explicit archive $A_{k}$, separate from the population. To emphasize non-dominated individuals, SPEA 2 uses a combination of the dominance count and the dominance rank methods. Each individual is assigned a raw fitness value depending on both the number of individuals it dominates and the number of individuals by which it is dominated. The density information is expressed as a function of the $k$-th smallest Euclidean distance in the objective space to the $k$-th nearest neighbor. The non-dominated individuals from the union of the archive and the current population are then updated. In particular, if the number of non-dominated individuals is less than the pre-established archive size, some dominated individual from the current pool form part of the archive. Otherwise, some individuals are removed from the archive using a truncation operator. This procedure recursively removes individuals based on the nearest neighbor Euclidean distance. If there is more than one candidate solution with the same minimum distance, then the decision is made by considering the second nearest neighbor, and so forth. The mating pool used to generate the next population $P_{t+1}$ is filled by 
the individuals of the updated archive selected on the basis of a given selection mechanism. The offsprings are then generated by a set of variation operators as in the algorithm NSGA-II.



\section{Solution approaches}

NSGA-II and SPEA 2 cannot be directly implemented in their standard format for the solution of real-world portfolio selection problems and special treatments need to be adopted in relation to a number of issues, like the encoding type, genetic operators, and constraint-handling procedures.

\section{Solution representation and initialization}

We represent a portfolio by a real vector of size $n$, denoted by $\mathbf{x}=\left(x_{1}, \ldots, x_{n}\right)^{T} \in \mathbb{R}^{n}$, where $x_{i}$ is the proportion of budget invested in asset $i$, with $i=1, \ldots, n$. Each candidate solution in the initial population is generated by following Rubistein's (1982) procedure, which consists of the following steps:

i) generate $n$ independent unit-exponential random variables $Y_{1}, \ldots, Y_{n}$ and compute $T_{n}=\sum_{i=1}^{n} Y_{i}$

ii) define $x_{i}=Y_{i} / T_{n}$.

It is evident that, in this manner, all the individuals in the initial population are feasible.

\section{Reproduction process}

Two variants of the reproduction process are compared in this study, both employing a selection procedure, a crossover, and a mutation operator.

The first configuration is based on that proposed by (Anagnostopoulos and Mamanis 2011a) ${ }^{3}$.

1. Binary tournament selection is used for selecting the parents. In this process, two individuals are chosen randomly from the population and compete against each 
other. The individual with the highest fitness wins and is included as one parent for the next steps of the reproduction.

2. The uniform crossover operator is then applied to the population of parents to produce the offspring population. Two parents generate a single child and its value for each array is selected with equal probability from one or another parent.

3. The set of children is finally subject to the Gaussian mutation. A percentage $P_{m u t}$ of these individuals is selected. Then, each member $\mathbf{x}$ of this subset has a probability $\mu_{m}$ that a gene $x_{i}$ mutates according to the following rule:

$$
x_{i}^{\prime \prime}= \begin{cases}x_{i}+\sigma_{m} \text { rand }_{1}, & \text { if } \text { rand }_{0}<\mu_{m}, \\ x_{i}, & \text { otherwise. }\end{cases}
$$

where $\sigma_{m}$ is the mutation step size, and $\operatorname{rand}_{0}$, rand $d_{1}$ represent two independent random numbers from a standard normal distribution, with $i=1, \ldots, n$.

In the numerical comparisons, the algorithms using this set up are denoted by NSGA-IIb and SPEA 2b. The alternative we propose is as follows.

1. Uniform selection is used to generate two sub-populations of parents. The first of these sets contains the fraction $P_{\text {cross }}$ of the original population that is involved into the recombination step. The second set contains the fraction $P_{m u t}$ of the original population that is subject to mutation. These sets cannot be separated, in that the same individual can enter the crossover stage and can mutate.

2. The intermediate crossover is applied to the first sub-population of parents to generate a first set of offsprings. Differently from the uniform crossover, this operator generates two children for each pair of parents $\mathbf{x}_{1}, \mathbf{x}_{2}$, as follows

$$
\begin{aligned}
& x_{1 i}^{\prime}=c s f_{i} x_{1 i}-\left(1-c s f_{i}\right) x_{2 i} \\
& x_{2 i}^{\prime}=c s f_{i} x_{2 i}-\left(1-c s f_{i}\right) x_{1 i}
\end{aligned}
$$

where $c s f_{i}$, called the crossover scaling factor, is a random number in the interval $[-d, 1+d], d \in \mathbb{R}$ is a parameter to be tuned, and $\mathbf{x}_{1}^{\prime}, \mathbf{x}_{2}^{\prime}$ are the associated children, with $i=1, \ldots, n$. The second population of parents is modified by the Gaussian mutation as in the first configuration.

3. The sub-populations of children are finally gathered together to form the offspring population.

The variants of the considered MOEAs involving this reproduction design are denoted by NSGA-IIa and SPEA 2a.

\section{Constraint-handling procedure}

There are several techniques proposed to handle constraints in MOEAs (see MezuraMontes and Coello (2011) and references therein). Since the feasible set in our portfolio optimization problems is represented by the unitary simplex (1), the best procedure is to use the following two-step repair mechanism (Liagkouras and Metaxiotis 2015).

(a) Each candidate solution $\mathbf{x} \in \mathbb{R}^{n}$ is first clamped by projecting it onto $[0,1]^{n}$ :

$$
\tilde{x}_{i}= \begin{cases}0, & \text { if } x_{i}<0 \\ 1, & \text { if } x_{i}>1 \\ x_{i}, & \text { otherwise }\end{cases}
$$


with $i=1, \ldots, n$. In this manner, $\widetilde{\mathbf{x}}=\left(\widetilde{x}_{1}, \ldots, \widetilde{x}_{n}\right)^{T}$ satisfies the lower bound constraints.

(b) The projected vector $\widetilde{\mathbf{x}}$ is then normalized through the transformation

$$
\widetilde{\widetilde{x}}_{i}=\frac{\tilde{x}_{i}}{\sum_{j=1}^{n} \widetilde{x}_{j}}, \quad i=1, \ldots, n .
$$

After this step, the individual $\widetilde{\mathbf{x}}=\left(\widetilde{\widetilde{x}}_{1}, \ldots, \widetilde{\widetilde{x}}_{n}\right)^{T}$ also verifies the budget constraint.

This procedure makes all the individuals involved in the search feasible. The corresponding objective function values can now be computed and the MOEAs described above can be applied to identify an approximation of the Pareto set.

\section{Computational complexity}

Let us assume that the size of the population and the size of the external archive are equal to $N$, the number of objectives is $m$, the number of generations is $G$, and the dimension of the vector of decision variables is $n$. Based on the analysis presented by (Deb et al. 2002) for one generation, and taking into account the different definition of the parent population for the two configurations, the NSGA-IIb algorithm requires $\mathcal{O}\left(m(2 N)^{2}\right)$ computations while the NSGA-IIa algorithm has a running time bounded by $\mathcal{O}\left(m\left(1+2 P_{\text {cross }}+P_{\text {mut }}\right)^{2} N^{2}\right)$. In a similar manner, the time complexity of SPEA $2 \mathrm{~b}$ is $\mathcal{O}\left((2 N)^{3}\right)$ and that of SPEA 2 a is $\mathcal{O}\left(\left(1+2 P_{\text {cross }}+P_{\text {mut }}\right)^{3} N^{3}\right)$ for each generation. Both the procedures used for the selection step require $\mathcal{O}(N)$ computations. The uniform crossover operator runs in time $\mathcal{O}(N D)$, while the intermediate crossover needs time $\mathcal{O}\left(2 P_{\text {cross }} N D\right)$, since it is applied to a fraction $P_{\text {cross }}$ of the individuals and generate two offsprings for each pair of parents. The Gaussian mutations can be undertaken in time $\mathcal{O}(N D)$ for the first configuration and in time $\mathcal{O}\left(P_{\text {mut }} N D\right)$ for the second one. Table 1 summarizes the worst run-time complexities of the algorithms treated in this section in $G$ generations.

\section{Experimental analysis}

In this section, we assess the effectiveness of the two variants of NSGA-II and SPEA 2 in solving the proposed instances of the portfolio optimization problem.

\section{Description of the datasets}

The experiments are based on five public datasets provided by (Bruni et al. 2016). They include the weekly linear returns of the constituents of the following stock markets: Dow Jones Industrial Average, Fama-French Market Industry, NASDAQ 100, S\&P 500, and NASDAQ Composite. As shown in Table 2, the test problems range from 28 assets for the smaller case study to 1203 assets for the bigger one.

Table 1 Run time complexity for the algorithms

\begin{tabular}{ll}
\hline Algorithm & Run-time \\
\hline NSGA-Ilb & $\mathcal{O}\left(G m(2 N)^{2}\right)$ \\
NSGA-lla & $\mathcal{O}\left(G m\left(1+2 P_{\text {cross }}+P_{\text {mut }}\right)^{2} N^{2}\right)$ \\
SPEA2b & $\mathcal{O}\left(G(2 N)^{3}\right)$ \\
SPEA 2a & $\mathcal{O}\left(G\left(1+2 P_{\text {cross }}+P_{\text {mut }}\right)^{3} N^{3}\right)$ \\
\hline
\end{tabular}


Table 2 Datasets of weekly returns considered in this study (Bruni et al. 2016)

\begin{tabular}{lllll}
\hline Dataset name & Stock market index & Assets $(n)$ & Time interval & \# of obs. \\
\hline DowJones & Dow Jones Industrial Average & 28 & Feb 1990 - Apr 2016 & 1363 \\
FF49Industries & Fama and French 49 Industry & 49 & Jul 1969- Jul 2015 & 2325 \\
NASDAQ100 & NASDAQ 100 & 82 & Nov 2004 - Apr 2016 & 596 \\
SP500 & S\&P 500 & 442 & Nov 2004 - Apr 2016 & 595 \\
NASDAQComp & NASDAQ Composite & 1203 & Feb 2003 - Apr 2016 & 685 \\
\hline
\end{tabular}

From Table 3, it can be pointed out that, in all the datasets, an asset with a moderately or highly negative skewed return distribution (Skew $<-0.5$ ) presents a value of the standard semi-deviation (Stsd), that is, the squared root of the semi-variance, which is higher than the corresponding value of the standard deviation (Std). Conversely, an asset with a moderately or highly positive skewed return distribution (Skew $>0.5$ ) has a Stsd value less than the value of the Std. Assets with a skewness between -0.5 and 0.5 present approximately symmetric return distributions, resulting in very similar Stsd and Std values.

Consequently, loss-averse investors can use standard semideviation (or, equivalently, semi-variance) to capture the downside risk conveyed in skewness properly. In this sense, the portfolio selection models designed in the previous section are more appealing than the mean variance model for acting investment choices.

\section{Performance metrics and statistical testing}

It is non-trivial to evaluate the quality of the solution sets of MOEAs for two main reasons. First, the presence of multiple conflicting goals makes the definition of a "better algorithm" vague. Second, the stochastic nature of these optimizers suggests that comparisons based on the approximation sets from a single run of each algorithm are not correct.

Regarding the first problem, we can identify three major performance criteria in multiobjective optimization: the capacity of a given algorithm to generate an appropriate number of non-dominated solutions, the convergence of the solution set to the true Pareto front, and the diversity of the solutions in the objective space. Accordingly, optimal solution sets with a large number of non-dominated solutions, approaching the true Pareto front and even scattering are generally desirable (Jiang et al. 2014).

The metrics proposed in the literature usually synthesize the information content of the solution set by considering only one or two criteria at a time. Therefore, it is advisable to employ a combination of different performance metrics in order to provide a complete analysis of the experimental results. In this sense, we consider the following four metrics.

Table 3 Classification of the assets belonging to each dataset in terms of skewness (Skew), standard semideviation (Stsd) and standard deviation (Std)

\begin{tabular}{|c|c|c|c|c|}
\hline \multirow[b]{2}{*}{ Dataset name } & \multicolumn{2}{|c|}{ Skew $<-0.5$} & \multicolumn{2}{|c|}{ Skew $>0.5$} \\
\hline & Assets & Stsd $>$ Std & Assets & Stsd $<$ Std \\
\hline DowJones & 2 & 2 & 1 & 1 \\
\hline FF49Industries & 2 & 2 & 6 & 6 \\
\hline NASDAQ100 & 2 & 2 & 20 & 20 \\
\hline SP500 & 25 & 25 & 109 & 108 \\
\hline NASDAQComp & 19 & 15 & 668 & 668 \\
\hline
\end{tabular}


(i) Schott's spacing metric (S) (Schott 1995)

The spacing metric measures how evenly the solutions are distributed in the approximate efficient front $A$ and it is expressed as

$$
S(A)=\sqrt{\frac{1}{|A|} \sum_{i=1}^{|A|}\left(d_{i}-\bar{d}\right)^{2}}
$$

where $d_{i}$ represents the minimum value of the sum of the absolute difference in objective function values between the $i$-th solution and any other solution in the obtained non-dominated set and $\bar{d}$ is the mean value of these distance measures. When the solutions are equidistantly spaced, the corresponding distance measure is small. Thus, an algorithm finding a set of non-dominated solutions with smaller spacing is better.

(ii) Generalized spread metric $\left(\Delta^{*}\right)$ (Zhou et al. 2006)

The generalized spread metric is a generalization of the well-known $\Delta$ metric of (Deb et al. 2002) and simultaneously gauges the distribution and spread of an optimal solution set $A$ for high dimensional multi-objective optimization problems. It takes the form:

$$
\Delta^{*}(A, P)=\frac{\sum_{m=1}^{M} d_{m}^{P}+\sum_{i=1}^{|A|}\left|d_{i}-\bar{d}\right|}{\sum_{m=1}^{M} d_{m}^{e}+|A| \bar{d}}
$$

where $d_{i}$ denotes the Euclidean distance between neighboring solutions with the mean value $\bar{d}$. The term $d_{m}^{P}$ is the distance between the extreme (bounding) solutions of $A$ and of the true Pareto front $P$ corresponding to the $m$-th objective function.

An algorithm finding a smaller value of $\Delta^{*}$ generates a better uniformly distributed set of non-dominated solutions.

(iii) Inverted generation distance (IGD) (Zhang and Li 2007)

The IGD index has the following formulation

$$
\operatorname{IGD}(A, P)=\frac{\sqrt{\sum_{i=1}^{|P|} d_{i}^{2}}}{|P|}
$$

where $d_{i}$ is the Euclidean distance (in the objective space) between the $i$-th member of the true Pareto front $P$ and the closest solution in $A$. If $|P|$ is large enough to represent the Pareto front very well, $\operatorname{IGD}(A, P)$ could measure both the diversity and convergence of $A$.

A low value of $\operatorname{IGD}(A, P)$ means $A$ is very close to the Pareto front and does not miss any part of it. Thus, an algorithm with a lower value of IGD is better.

(iv) Hypervolume (HV) (Zitzler and Thiele 1999)

Similar to the IGD index, the hypervolume indicator evaluates both diversity and convergence of an approximation set $A$. It is defined as the size of the portion of objective space that is dominated by at least one point of $A$ relative to a reference set $R$, which is formed by points worse than (or equal to) every point in $A$ in every objective. Formally, we define it as

$$
H V(A, R)=\text { volume }\left(\bigcup_{i=1}^{|A|} v_{i}\right)
$$


where $v_{i}$ is the hypercube constructed with the reference set $R$ and the solution $a_{i} \in A$ as the diagonal corners.

Large values of $H V$ indicate the approximate solutions are closer to the true Pareto front and, at the same time, scattered more evenly in the objective space. Thus, an algorithm with a large value of the $H V$ metric is desirable.

The second question posed at the beginning of this section can be resolved by comparing the algorithms through a sample of approximation sets from multiple runs. In this manner, we obtain a sample of values for each performance metric and for each portfolio optimization problem. A rigorous comparison between the algorithms can then be performed based on non-parametric inference testing (Coello et al. 2007).

Since the true Pareto front is a (continuous) manifold in the decision space, we approximate it by a surrogate front, given by the non-dominated points from the union of the solution sets of all the algorithms for all the simulations. This set is used to calculate the $\Delta^{*}$ and IGD metrics. Furthermore, before conducting the performance analysis, we normalize each objective function value $F_{i}(\mathbf{x}), i=1, \ldots, p+q$, in (3) according to the following transformation

$$
\tilde{F}_{i}(\mathbf{x})=\frac{F_{i}(\mathbf{x})-F_{i}^{\text {min }}}{F_{i}^{\text {max }}-F_{i}^{\text {min }}}
$$

where $F_{i}^{\text {min }}$ and $F_{i}^{\text {max }}$ are the minimum and maximum values of the $i$-th objective function for the corresponding surrogate Pareto front. In this manner, the objectives contribute almost equally to each performance metric and the results from the different algorithms can be fairly compared.

\section{Parameter settings}

Let us assume that the distribution of historical returns acts as a good proxy of the returns faced over the next holding period. Then, the historical simulation method is used to compute the financial scenarios $r_{i s}$, with $i=1, \ldots, n$ and $s=1, \ldots, S$, as well as the co-semi-variance matrix $C^{-}$.

All the variants of NSGA-II and SPEA 2 use identical population and archive sizes of 250, and 400 iterations for all the experiments as in (Anagnostopoulos and Mamanis 2011a). For the remaining parameters, the algorithms NSGA-IIb and SPEA 2b use the values proposed by the same authors. Instead, for NSGA-IIa and SPEA 2a, the parameters are tuned over the DowJones, FF49Industries, and NASDAQ100 datasets for each of the three portfolio optimization problems, for a total of nine test problems. For each pair of parents the intermediate crossover produces two offsprings while the uniform crossover provides a single child, and thus, to make fair comparisons among algorithms, we select the percentage of crossover $P_{\text {cross }}$ from the set $\{0.35,0.45\}$ and the parameter $d$, related to the crossover scaling factor, from $\{0,0.5,1\}$. The mutation percentage $P_{m u t}$ varies in $\{0.3,0.5\}$, the mutation probability $\mu_{m}$ can assume a value in the set $\{0.1,0.3\}$, while the mutation step size $\sigma_{m}$ is checked in $\{0.1,0.15,0.2\}$. In this manner, there are 72 possible combinations of these parameters. All the experiments are conducted 20 times for each algorithm and the hypervolume values corresponding to the final populations are recorded. Finally, since we search for a parameter set up that is widely applicable in finding suitable approximation fronts for all the test problems, the best group of parameter settings is selected based on the average ranking of the Friedman test for the values of the $H V$ index over the 
simulations (Derrac et al. 2011). Tables 4 and 5 report the configurations with the highest average rankings for the proposed MOEAs.

Overall, the results show that the best choices are higher values for $P_{\text {cross }}$ and $d$ and, at the same time, lower values for $\mu_{m}$ and $\sigma_{m}$. However, SPEA 2a needs a larger sample of mutants to achieve the best rankings with respect to NSGA-IIa. The configurations in bold are the best parameter settings and are used in the experiments.

\section{Computational results and discussion}

We perform a multi-problem analysis in which the four MOEAs are tested on 15 optimization problems (the three instances of the portfolio selection problem over the five datasets introduced in Table 2). To check the robustness of the results, 20 simulations for each algorithm and for each test problem are used. The algorithms are implemented in MATLAB R2018b and the experiments are carried out on a $2.2 \mathrm{GHz}$ Intel Core i7 laptop with 4 GB RAM.

Table 6 reports the average number of nondominated solutions provided by the algorithms in the final approximated optimal sets, divided by problem and dataset involved. It can be noticed that including the proposed configuration in the reproduction process increases the search capabilities of both NSGA-II and SPEA 2 with respect to the standard configuration. In particular, NSGA-IIa and SPEA 2a can identify sets of optimal solutions with a size almost equal to the pre-defined threshold of 250 individuals in all the test problems, while NSGA-IIb and SPEA 2b produce much smaller optimal sets as the complexity of the problems increases.

Tables 7, 8 and 9 focus on the distribution of the values for the performance metrics, averaged over the 20 simulations for each portfolio optimization problem. Overall, the superiority of NSGA-IIa and SPEA 2a is evident with respect to all the criteria used in the comparisons. Regarding the $S$ metric, for small- to medium-sized optimization problems, NSGA-IIa produces approximate efficient fronts with the smallest spacing. However, as the dimension of the problems increases, the standard versions of the algorithms become competitive. In terms of the generalized spread metric, NSGA-IIa confirms its good performance while SPEA 2a guarantees a better uniformly distributed set of non-dominated solutions for large-scale problems. Similar analysis can be undertaken for the inverted generation distance and for the hypervolume indicator. In these cases, NSGA-IIa and SPEA 2a are the best choices, and SPEA 2a is preferred for large-scale problems.

We check whether the differences in the performance are significant by using the $\mathrm{R}$ package called "scmamp" (Calvo and Santafé 2016). First, we apply the Friedman aligned omnibus test to detect if at least one of the algorithms performs differently to the others. The results reported in Table 10 show that the differences are significant for three out of

Table 4 Average rankings achieved by the Friedman test for the NSGA-Ila algorithm with different parameter settings

\begin{tabular}{lc}
\hline NSGA-lla & Rankings \\
\hline$P_{\text {cross }}=0.45, d=0.5, P_{\text {mut }}=0.5, \mu_{m}=0.1, \sigma_{m}=0.15$ & 58.89 \\
$\mathbf{P}_{\text {cross }}=\mathbf{0 . 4 5}, \mathbf{d}=\mathbf{1}, \mathbf{P}_{\text {mut }}=\mathbf{0 . 3}, \mu_{\mathbf{m}}=\mathbf{0 . 1}, \sigma_{\mathbf{m}}=\mathbf{0 . 1 0}$ & $\mathbf{5 9 . 3 3}$ \\
$P_{\text {cross }}=0.45, d=1, P_{\text {mut }}=0.3, \mu_{m}=0.1, \sigma_{m}=0.15$ & 58.00 \\
$P_{\text {cross }}=0.45, d=1, P_{\text {mut }}=0.3, \mu_{m}=0.3, \sigma_{m}=0.10$ & 57.89 \\
$P_{\text {cross }}=0.45, d=1, P_{\text {mut }}=0.5, \mu_{m}=0.3, \sigma_{m}=0.20$ & 59.22 \\
\hline The highest ranking is shown in bold &
\end{tabular}


Table 5 Average rankings achieved by the Friedman test for the SPEA 2a algorithm with different parameter settings

\begin{tabular}{lc}
\hline SPEA 2a & Rankings \\
\hline$P_{\text {cross }}=0.45, d=1, P_{\text {mut }}=0.3, \mu_{m}=0.1, \sigma_{m}=0.10$ & 64.89 \\
$P_{\text {cross }}=0.45, d=1, P_{\text {mut }}=0.3, \mu_{m}=0.1, \sigma_{m}=0.20$ & 63.44 \\
$\mathbf{P}_{\text {cross }}=\mathbf{0 . 4 5}, \mathbf{d}=\mathbf{1}, \mathbf{P}_{\mathbf{m u t}}=\mathbf{0 . 5}, \mu_{\mathbf{m}}=\mathbf{0 . 1}, \sigma_{\mathbf{m}}=\mathbf{0 . 1 0}$ & $\mathbf{6 9 . 2 2}$ \\
$P_{\text {cross }}=0.45, d=1, P_{\text {mut }}=0.5, \mu_{m}=0.1, \sigma_{m}=0.15$ & 67.89 \\
$P_{\text {cross }}=0.45, d=1, P_{\text {mut }}=0.5, \mu_{m}=0.1, \sigma_{m}=0.20$ & 67.56 \\
\hline aThe highest ranking is shown in bold &
\end{tabular}

four criteria. The algorithms have comparable performance only for the spacing metric. Thus, the analysis can proceed to characterize the differences found for the $\Delta^{*}, I G D$, and $H V$ indicators using a post-hoc procedure. As suggested in (Derrac et al. 2011), we use the Friedman aligned post-hoc test with the correction of (Bergmann and Hommel 1988). Table 11 lists the five hypotheses of equality among the four algorithms and the corresponding adjusted $p$-values. With a $1 \%$ level of significance, we find an improvement in the performance of NSGA-II and SPEA 2 when they involve the second selection and reproduction scheme.

The contrast between the medians of the samples of the results is finally computed considering all the pairwise comparisons (Garcia et al. 2010). This test points out the quantitative difference between two algorithms over multiple test problems, allowing us to estimate how much an algorithm outperforms another one. Table 12 lists the comparisons of the proposed algorithms, divided by the performance metric used. Notably, an estimator with a negative value for $\Delta^{*}$ and $I G D$, and a positive value for $H V$ indicates that the algorithm in that row outperforms the algorithm in the corresponding column. The estimators highlight that SPEA 2a is the best performing algorithm, although the difference with NSGA-IIa is negligible.

Table 6 Average number of nondominated solutions in the approximated Pareto front (Mean) with the corresponding standard deviation (Std) for each algorithm

\begin{tabular}{|c|c|c|c|c|c|c|c|c|c|}
\hline \multirow[t]{2}{*}{ Problem } & \multirow[t]{2}{*}{ Dataset } & \multicolumn{2}{|c|}{ NSGA-IIb } & \multicolumn{2}{|c|}{ NSGA-Ila } & \multicolumn{2}{|c|}{ SPEA 2b } & \multicolumn{2}{|c|}{ SPEA 2a } \\
\hline & & Mean & std & Mean & Std & Mean & Std & Mean & Std \\
\hline \multirow[t]{5}{*}{ Mean-SV } & DowJones & 52.10 & 5.75 & 248.06 & 4.93 & 55.65 & 6.12 & 250 & 0 \\
\hline & FF49Industries & 16.65 & 4.61 & 250 & 0 & 15.20 & 3.68 & 250 & 0 \\
\hline & NASDAQ100 & 14.25 & 2.90 & 250 & 0 & 13.40 & 2.89 & 250 & 0 \\
\hline & SP500 & 11.55 & 3.22 & 250 & 0 & 11.50 & 3.32 & 250 & 0 \\
\hline & NASDAQComp & 13.60 & 2.93 & 202.50 & 20.97 & 13.60 & 2.93 & 245.20 & 9.29 \\
\hline \multirow[t]{5}{*}{ Mean-CVaR } & DowJones & 49.70 & 6.24 & 247.33 & 4.86 & 51.15 & 6.66 & 250 & 0 \\
\hline & FF49Industries & 16.10 & 3.80 & 249.95 & 0.22 & 17.25 & 3.19 & 250 & 0 \\
\hline & NASDAQ100 & 16.45 & 3.27 & 250 & 0 & 16.80 & 3.86 & 250 & 0 \\
\hline & SP500 & 12.60 & 2.84 & 250 & 0 & 12.45 & 2.68 & 250 & 0 \\
\hline & NASDAQComp & 15.80 & 3.43 & 238.60 & 13.18 & 16.10 & 3.91 & 249.05 & 2.21 \\
\hline \multirow[t]{5}{*}{ Mean-CVaR-SV } & DowJones & 92.90 & 14.62 & 246.33 & 7.57 & 91.75 & 11.96 & 250 & 0 \\
\hline & FF49|ndustries & 22.25 & 7.04 & 250 & 0 & 21.25 & 6.70 & 250 & 0 \\
\hline & NASDAQ100 & 22.45 & 5.82 & 250 & 0 & 22.50 & 3.90 & 250 & 0 \\
\hline & SP500 & 18.20 & 6.18 & 250 & 0 & 18.85 & 7.51 & 250 & 0 \\
\hline & NASDAQComp & 25.65 & 6.03 & 250 & 0 & 25.65 & 5.59 & 250 & 0 \\
\hline
\end{tabular}




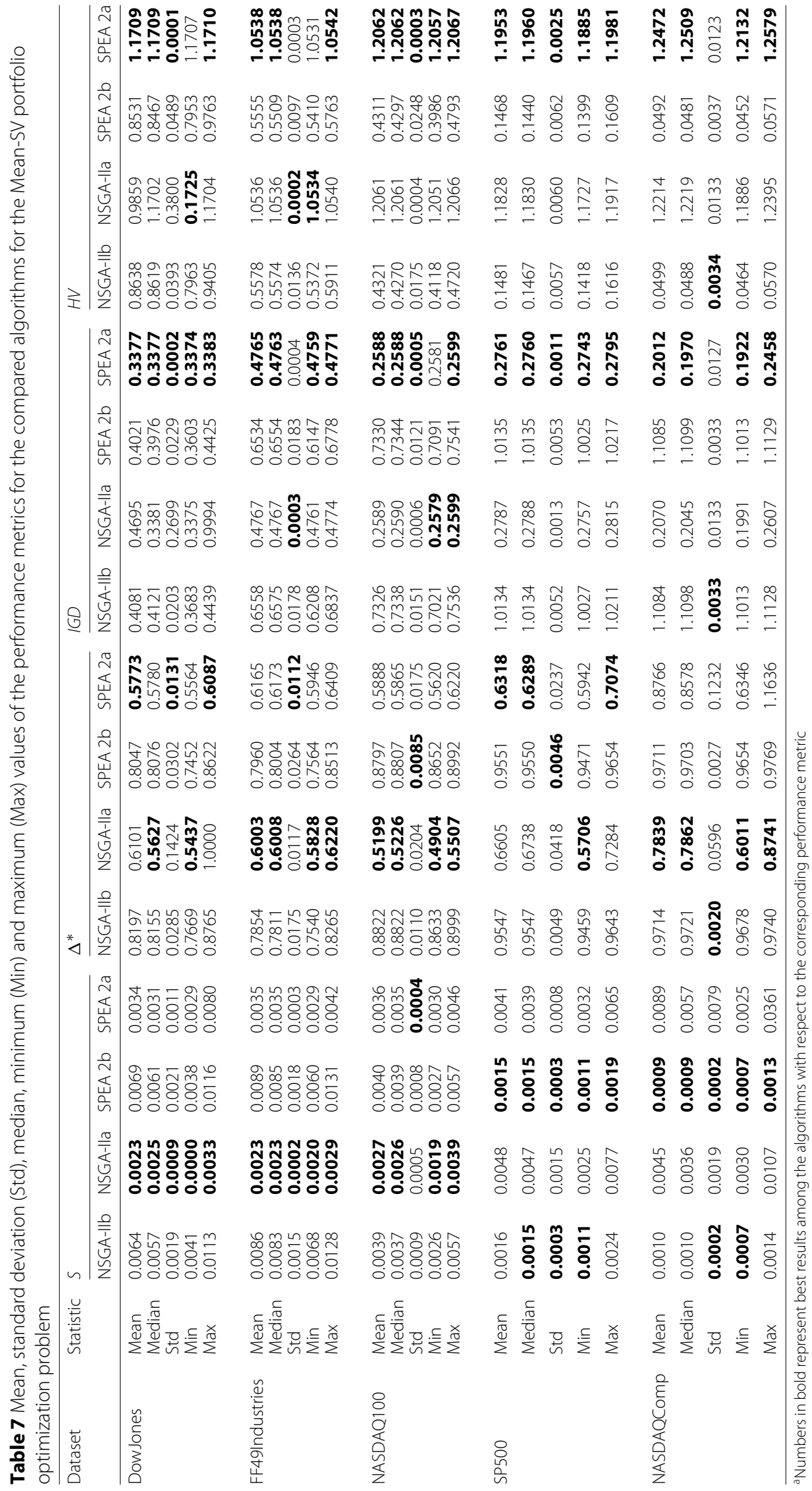




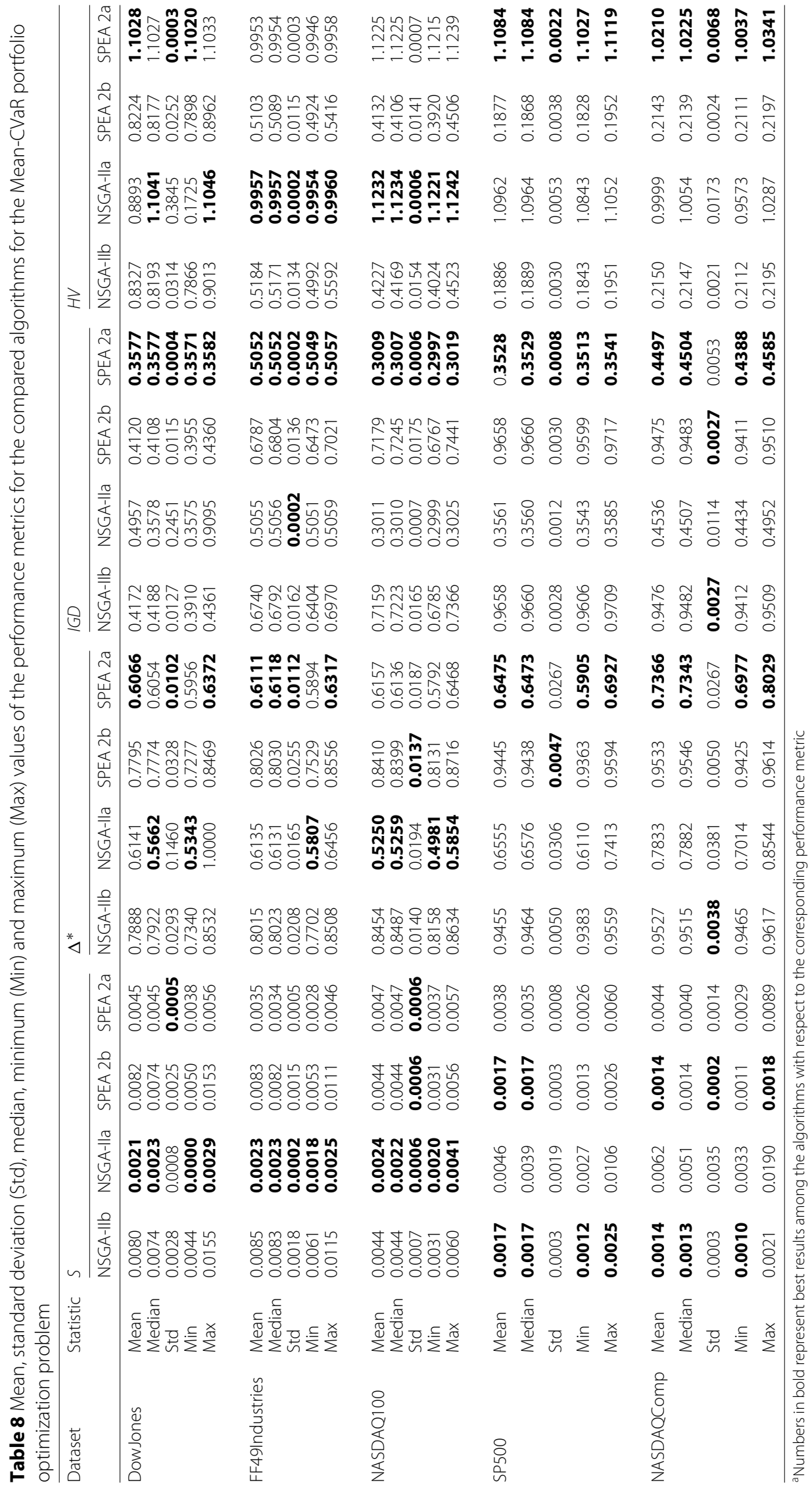




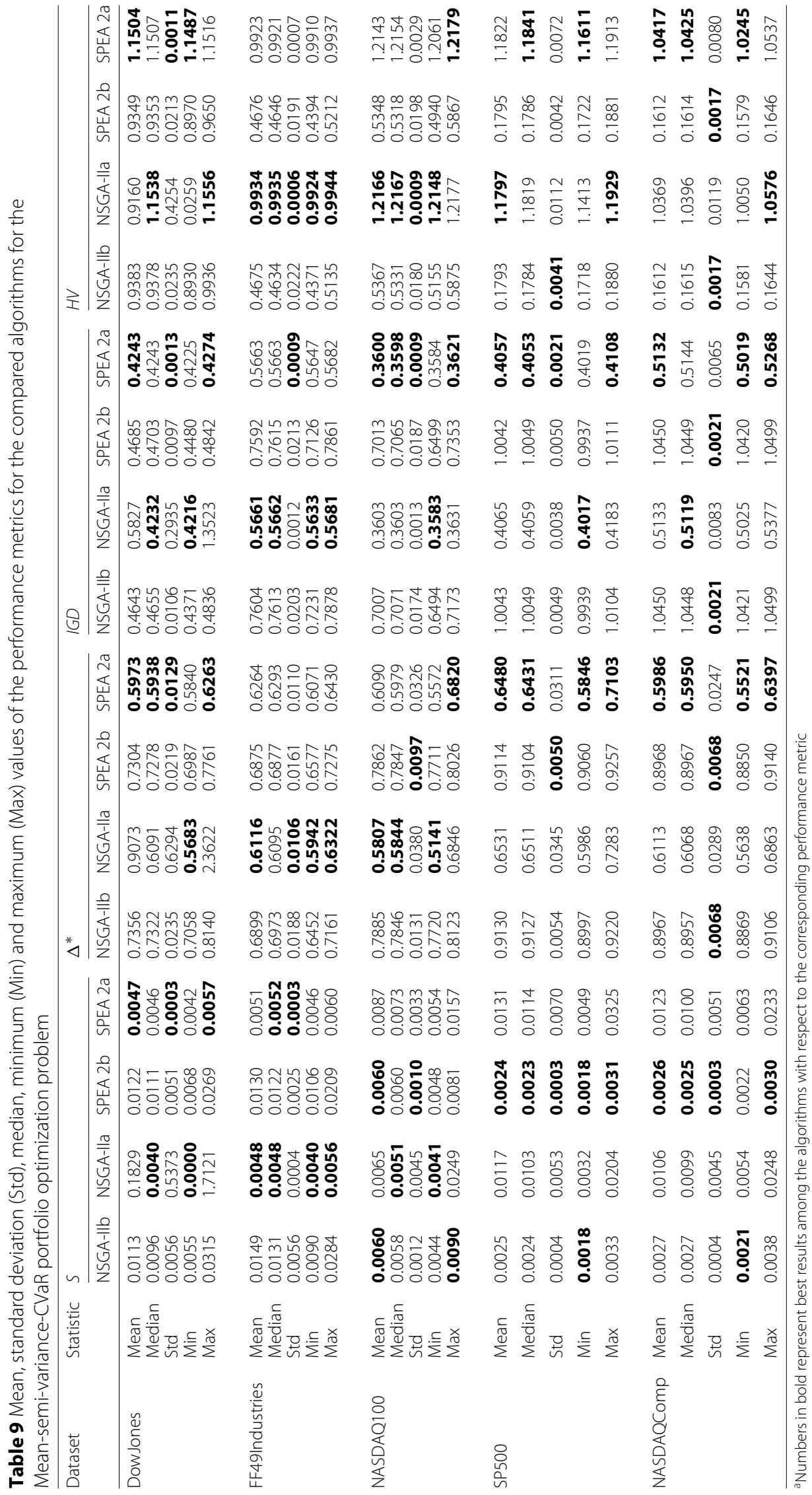


Table 10 Statistics and related $p$-values for the Friedman aligned omnibus test for each of the four performance metrics

\begin{tabular}{lll}
\hline Performance metric & Statistic & $p$-value \\
\hline$S$ & 0.443 & 0.9312 \\
$\Delta^{*}$ & 29.59 & $1.681 e-06$ \\
IGD & 30.42 & $1.124 e-06$ \\
$H V$ & 33.5 & $2.528 e-07$ \\
\hline
\end{tabular}

For illustrative purposes, Figs. 1 and 2 show a comparison of the Pareto fronts obtained by the four algorithms analyzed for the DowJones, NASDAQ100, and NASDAQComposite datasets corresponding to the simulations that attain the highest values of the hypervolume metric. The proposed versions of the algorithms NSGA-II and SPEA 2 can identify frontiers with more evenly scattered and spread out points than the standard alternatives in all the test problems. The differences among the algorithms are already marked for problems with 83 decision variables. As the size of the investible universe increases, NSGA-IIb and SPEA 2b no longer cover the shape of the Pareto front and tend to produce highly inefficient solutions, with higher risks and lower expected returns. Moreover, for the three-objective portfolio optimization problem, the charts on the left of Fig. 2 highlight the relationship between Stsd and CVaR, which is almost linear for higher values of risk.

Figure 3 displays the mean processing time of the four algorithms for each portfolio model and for each dataset. The procedures implementing the proposed configuration demand more computational resources owing to the use of larger offspring populations. The difference becomes more evident as the number of decision variables increases. Moreover, the total run time is greatly influenced by the time spent evaluating the CVaR objective function. The time spent on the management of the archive for the SPEA 2 algorithms is another cost to take into account.

In summary, balancing the results from the statistical analysis and those concerning the computational complexity, we suggest that the NSGA-II algorithm with the proposed configuration should be used to solve the portfolio optimization problems involving downside risk measures. In fact, it produces approximated sets similar to the SPEA 2a algorithm but takes less time to do so.

\section{Concluding remarks and future research}

In this study, we described three instances of the portfolio selection problem designed to handle the downside risk of an investment properly. A flexible multi-objective reward-torisk framework was presented in which expected returns, semi-variance, and CVaR of a

Table 11 Adjusted $p$-values for the Friedman aligned post-hoc test with Bergmann and Hommel's correction for multiple comparisons among the four algorithms

\begin{tabular}{llll}
\hline Hypothesis & \multicolumn{2}{l}{ Adj. $p$-values } & HV \\
\cline { 2 - 4 } & $\Delta^{*}$ & $6.889 e-05$ & $9.857 e-06$ \\
\hline NSGA-IIb versus NSGA-Ila & $6.276 e-05$ & 1 & 1 \\
NSGA-IIb versus SPEA 2b & 1 & $1.692 e-05$ & $8.199 e-06$ \\
NSGA-IIb versus SPEA 2a & $3.098 e-05$ & 1 & 1 \\
NSGA-Ila versus SPEA 2a & 1 & $1.692 e-05$ & $8.199 e-06$ \\
SPEA 2b versus SPEA 2a & $3.098 e-05$ &
\end{tabular}


Table 12 Contrast estimation results for the performance metric

\begin{tabular}{llllll}
\hline Performance metric & Estimation & NSGA-llb & NSGA-lla & SPEA 2b & SPEA 2a \\
\hline$\Delta^{*}$ & NSGA-llb & 0 & 0.206 & 0.0037 & 0.2158 \\
& NSGA-lla & -0.206 & 0 & -0.2023 & 0.0098 \\
& SPEA 2b & -0.0037 & 0.2023 & 0 & 0.2121 \\
SPEA 2a & -0.2158 & -0.0098 & -0.2121 & 0 \\
NSD & NSGIllb & 0 & 0.4151 & -0.0001 & 0.4157 \\
& NSGAlla & -0.4151 & 0 & -0.4161 & 0.0006 \\
SPEA 2b & 0.0001 & 0.4161 & 0 & 0.4167 \\
HV & SPEA 2a & -0.4157 & -0.0006 & -0.4167 & 0 \\
& NSGA-llb & 0 & -0.7012 & 0.0054 & -0.7032 \\
& NSGA-lla & 0.7012 & 0 & 0.7066 & -0.002 \\
& SPEA 2b & -0.0054 & -0.7066 & 0 & -0.7086 \\
& SPEA 2a & 0.7032 & 0.002 & 0.7086 & 0 \\
\hline
\end{tabular}
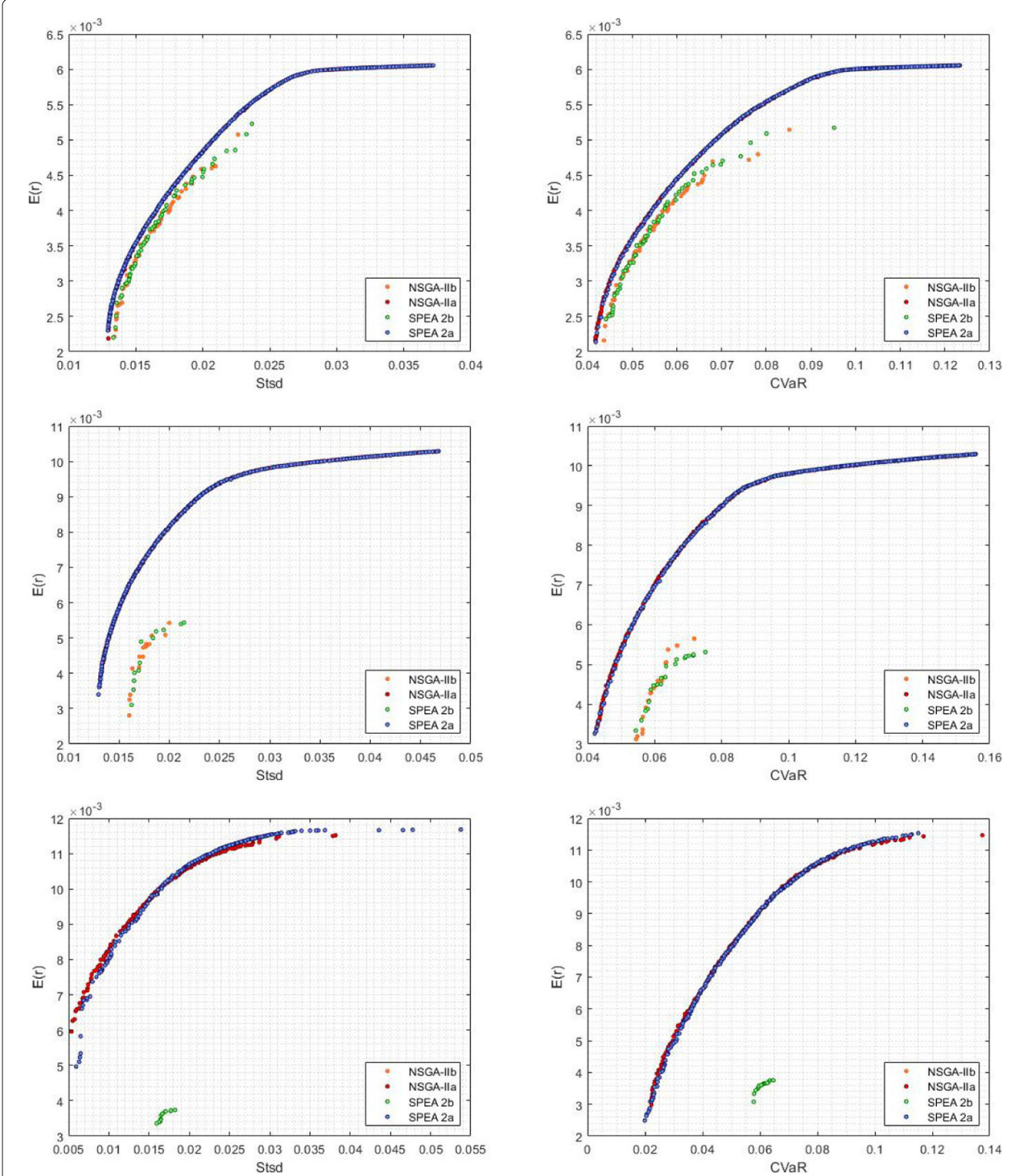

Fig. 1 Approximated Pareto fronts for the Mean-semi-variance (on the left) and Mean-CVaR (on the right) portfolio optimization problems corresponding to the simulations with the highest $H V$ values obtained by NSGA-Ilb, NSGA-lla, SPEA 2b and SPEA 2a for the datasets DowJones (charts at the top), NASDAQ100 (charts in the middle) and NASDAQComposite (charts below) 


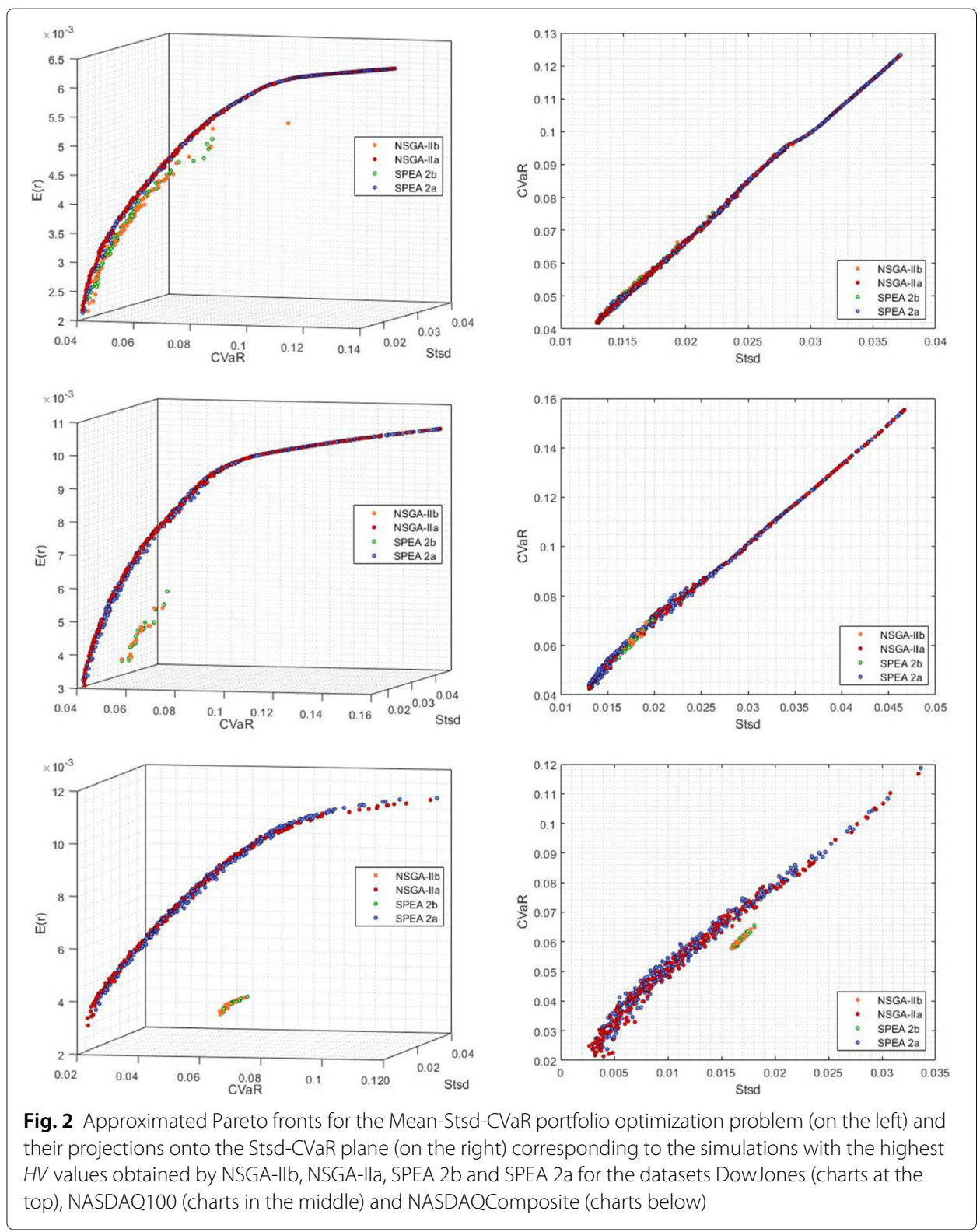

portfolio can be optimized simultaneously. These problems were tackled using two nondominated sorting algorithms, namely, NSGA-II and SPEA 2, which have already showed competitive performance for the mean variance problem. In particular, we proposed a novel combination of operators for the selection and reproduction phases to be included in both algorithms. A comparative analysis was undertaken with respect to a second variant of the same algorithms, involving another configuration design. We used five publicly available datasets ranging from small- to large-sized portfolio optimization problems. The capabilities of the procedures were assessed in terms of four performance metrics. Finally, a set of statistical tests checked the robustness of these findings. Overall, the numerical experiments showed that the proposed algorithms outperformed the others with respect to all the criteria. Even if the algorithms with the novel variation configuration demanded the use of more computational time as the dimension of the problems increased, they 

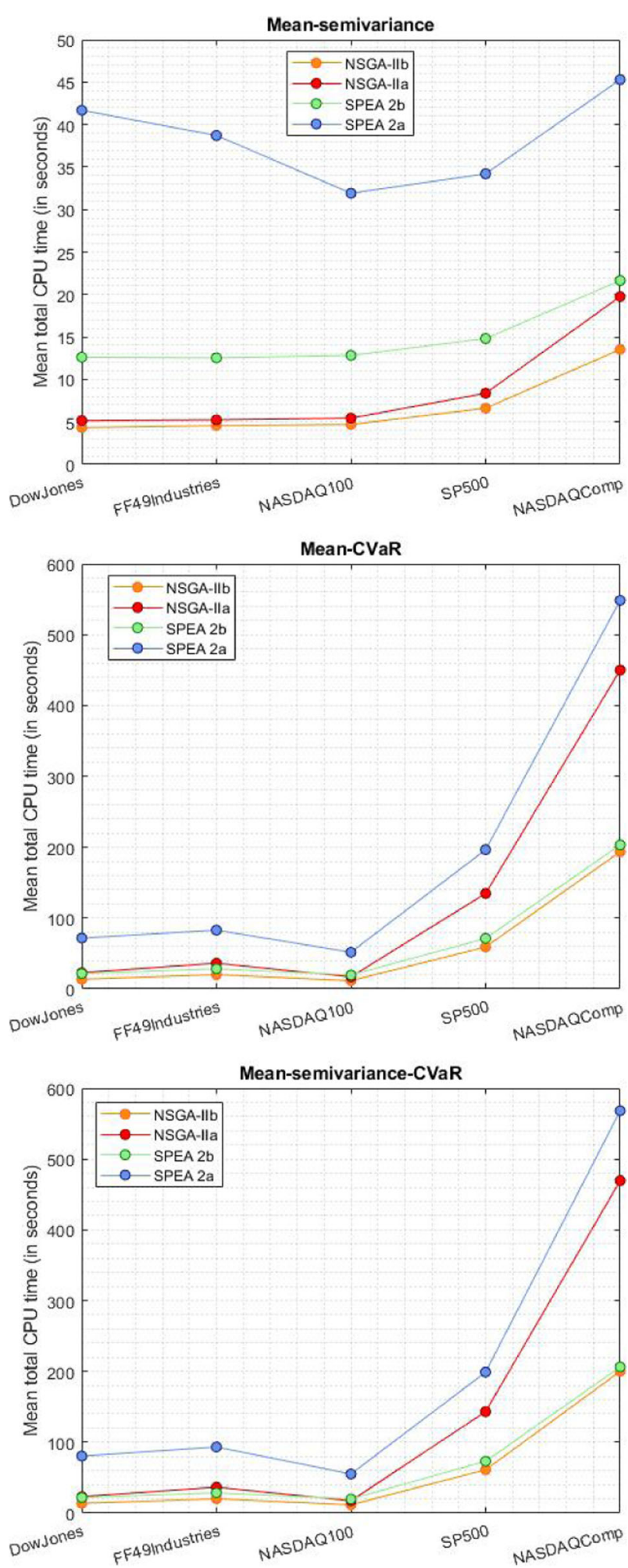

Fig. 3 Mean total CPU times (in seconds) for the Mean-semi-variance (charts at the top), Mean-CVaR (charts in the middle) and Mean-semi-variance-CVaR (charts below) portfolio optimization problems for 100,000 function evaluations of the algorithms NSGA-Ilb, NSGA-Ila, SPEA 2b and SPEA 2a for the considered datasets over 20 simulations 
nonetheless yielded reasonable results for the cases in which the other algorithms failed to capture the shape of the Pareto front properly.

These research findings can be put in practice to improve the risk management infrastructure of an investment company. The inclusion of several risk measures in the portfolio optimization process can increase the capabilities of the system to describe the risk, providing more attractive investment opportunities.

Future research work on the topic includes the analysis of out-of-sample effectiveness for this type of strategy, which is expected to be improved by the incorporation of other simulation techniques to estimate semi-variance and CVaR. We are also interested in exploring mechanisms that adaptively exploit several selection schemes and reproduction operators to accelerate convergence and allow the search to be stopped automatically when a suitable level of quality for the approximated set has been attained.

\section{Endnotes}

${ }^{1}$ Under these assumptions, the distribution function $F_{R(\mathbf{x})}$ is such that no jumps and no flat parts occur, implying that the equation $F_{R(\mathbf{x})}(z)=\alpha$ has a unique solution for any $\alpha \in(0,1)$, say $z^{*}=F_{R(\mathbf{x})}^{-1}(\alpha)$, where $F_{R(\mathbf{x})}^{-1}$ denotes the inverse of $F_{R(\mathbf{x})}$.

${ }^{2}$ The terms solution and individual will be used interchangeably, since individuals in the population represent solutions to the problem that is being optimized.

${ }^{3}$ The original study uses a mixed-integer solution encoding and two sets of variation operators to solve the mean variance optimization problem with cardinality constraints. In our paper, we only focus on the procedures concerning the real part of their encoding for the reproduction process.

\section{Abbreviations}

The following abbreviations are used in this paper:

$\Delta^{*}$ : Generalized spread metric; A: Approximate Pareto front; $C^{-}$: Co-semi-variance matrix; CVaR: Conditional value-at-risk; HV: Hypervolume; IGD: Inverted generation distance; MOEA: Multi-objective evolutionary algorith; NSGA-II: Non-dominated sorting genetic algorithm II; NSGA-Ila: Proposed version of the NSGA-II algorithm; NSGA-Ilb: Version of the NSGA-II algorithm with selection and reproduction as in (Anagnostopoulos and Mamanis 2011a); P: True Pareto front; S: Schott's spacing metric; SPEA 2: Strength Pareto evolutionary algorithm 2; SPEA 2a: Proposed version of the SPEA 2 algorithm; SPEA 2b: Version of the SPEA 2 algorithm with selection and reproduction as in (Anagnostopoulos and Mamanis 2011 a); Stsd: Standard semideviation; $V^{-}$: Semi-variance; VaR: Value-at-risk; $\mathcal{X}$ : Set of admissible portfolios

\section{Acknowledgments}

The authors would like to thank Gang Kou and Yong Shi for their assistance. The comments and suggestions of four reviewers are also gratefully acknowledged.

\section{Other declarations}

All the authors have approved the manuscript for submission and confirm that the content of the manuscript has not been published, or submitted for publication elsewhere.

\section{Authors' contributions}

All authors have equally contributed to this work and approved the final manuscript.

Funding

No funding was received for this research.

Availability of data and materials

Data have been downloaded from https://www.sciencedirect.com/science/article/pii/S2352340916303997?via\%3Dihub.

\section{Competing interests}

The authors declare that they have no competing interests.

\section{Author details}

${ }^{1}$ Department of Economics, Business, Mathematics and Statistics, University of Trieste, Piazzale Europa 1, 34127 Trieste, Italy. ${ }^{2}$ Department of Industrial Engineering, Faculty of Technology and Engineering, East of Guilan, University of Guilan, 44891-63157 Rasar-Vajargah, Iran. ${ }^{3}$ Department of Engineering Sciences, Faculty of Technology and Engineering, East of Guilan, University of Guilan, 44891-63157 Rasar-Vajargah, Iran. 
Received: 19 April 2018 Accepted: 26 May 2019

Published online: 05 June 2019

\section{References}

Acerbi C, Tasche D (2002) On the coherence of expected shortfall. J Bank Financ 26(7):1487-1503

Anagnostopoulos KP, Mamanis G (2011a) The mean-variance cardinality constrained portfolio optimization problem: An experimental evaluation of five multiobjective evolutionary algorithms. Expert Syst Appl 38(11):14208-14217

Anagnostopoulos KP, Mamanis G (2011b) Multiobjective evolutionary algorithms for complex portfolio optimization problems. Comput Manag Sci 8(3):259-279

Arnone S, Loraschi A, Tettamanzi A, et al (1993) A genetic approach to portfolio selection. Neural Netw World 3(6):597-604

Artzner P, Delbaen F, Eber J-M, Heath D (1999) Coherent measures of risk. Math Financ 9(3):203-228

Baixauli-Soler JS, Alfaro-Cid E, Fernández-Blanco MO (2010) Several risk measures in portfolio selection: Is it worthwhile? Span J Financ Acc/Rev Española de Financiación y Contabilidad 39(147):421-444

Baixauli-Soler JS, Alfaro-Cid E, Fernandez-Blanco MO (2012) A naïve approach to speed up portfolio optimization problem using a multiobjective genetic algorithm. Investigaciones Europeas de Dirección y Economía de la Empresa 18(2):126-131

Ballestero E, Pla-Santamaria D (2005) Grading the performance of market indicators with utility benchmarks selected from footsie: a 2000 case study. Appl Econ 37(18):2147-2160

Bergmann B, Hommel G (1988) Improvements of general multiple test procedures for redundant systems of hypotheses. In: Multiple Hypothesenprüfung/Multiple Hypotheses Testing. Springer, Berlin. pp 100-115

Bruni R, Cesarone F, Scozzari A, Tardella F (2016) Real-world datasets for portfolio selection and solutions of some stochastic dominance portfolio models. Data Brief 8:858-862

Calvo B, Santafé RG (2016) scmamp: Statistical comparison of multiple algorithms in multiple problems. R J 8(1):248-256

Chang T-J, Meade N, Beasley JE, Sharaiha YM (2000) Heuristics for cardinality constrained portfolio optimisation. Comput Oper Res 27(13):1271-1302

Chang T-J, Yang S-C, Chang K-J (2009) Portfolio optimization problems in different risk measures using genetic algorithm. Expert Syst Appl 36(7):10529-10537

Chao X, Kou G, Peng Y (2019) Behavior monitoring methods for trade-based money laundering integrating macro and micro prudential regulation: A case from china. Technol Econ Dev Econ. https://doi.org/10.3846/tede.2019.9383

Chen Y, Wang X (2015) A hybrid stock trading system using genetic network programming and mean conditional value-at-risk. Eur J Oper Res 240(3):861-871

Coello CAC, Lamont GB, Van Veldhuizen DA (2007) Evolutionary Algorithms for Solving Multi-objective Problems. Springer, New York

Crama Y, Schyns M (2003) Simulated annealing for complex portfolio selection problems. Eur J Oper Res 150(3):546-571

Cumova D, Nawrocki D (2011) A symmetric Ipm model for heuristic mean-semivariance analysis. J Econ Bus 63(3):217-236

Cura T (2009) Particle swarm optimization approach to portfolio optimization. Nonlinear Anal Real World Appl 10(4):2396-2406

Deb K, Pratap A, Agarwal S, Meyarivan T (2002) A fast and elitist multiobjective genetic algorithm: NSGA-II. IEEE Trans Evol Comput 6(2):182-197

Derrac J, García S, Molina D, Herrera F (2011) A practical tutorial on the use of nonparametric statistical tests as a methodology for comparing evolutionary and swarm intelligence algorithms. Swarm Evol Comput 1(1):3-18

Dueck G, Winker P (1992) New concepts and algorithms for portfolio choice. Appl Stoch Model Bus Ind 8(3):159-178

Eftekharian SE, Shojafar M, Shamshirband S (2017) 2-phase NSGA II: An optimized reward and risk measurements algorithm in portfolio optimization. Algorithms 10(4):130

Emmerich MT, Deutz AH (2018) A tutorial on multiobjective optimization: fundamentals and evolutionary methods. Nat Comput 17(3):585-609

Ergu D, Kou G, Shi Y, Shi Y (2014) Analytic network process in risk assessment and decision analysis. Comput Oper Res 42:58-74

Ertenlice O, Kalayci CB (2018) A survey of swarm intelligence for portfolio optimization: Algorithms and applications. Swarm Evol Comput 39:36-52

Estrada J (2008) Mean-semivaraince optimization: A heuristic approach. J Appl Financ 18(1):57-72

Fishburn PC (1977) Mean-risk analysis with risk associated with below-target returns. Am Econ Rev 67(2):116-126

Gaivoronski AA, Pflug G (2005) Value-at-risk in portfolio optimization: properties and computational approach. J Risk $7(2): 1-31$

Garcia S, Fernández A, Luengo J, Herrera F (2010) Advanced nonparametric tests for multiple comparisons in the design of experiments in computational intelligence and data mining: Experimental analysis of power. Inf Sci 180(10):2044-2064

Gen M, Cheng R (2000) Genetic Algorithms and Engineering Optimization, vol 7. Wiley, New York

Gilli M KE, Hysi H (2006) A data-driven optimization heuristic for downside risk minimization. J Risk 8(3):1-18

Guerard JB (2009) Handbook of Portfolio Construction: Contemporary Applications of Markowitz Techniques. Springer, New York

Hinterding R (1995) Gaussian mutation and self-adaption for numeric genetic algorithms. In: Evolutionary Computation, 1995., IEEE International Conference On, vol. 1. IEEE Press, Piscataway. pp 384-389

Hochreiter R (2007) An evolutionary computation approach to scenario-based risk-return portfolio optimization for general risk measures. In: Workshops on Applications of Evolutionary Computation. LNCS, vol. 4448. Springer, Heidelberg. pp 199-207

Hogan WW, Warren JM (1972) Computation of the efficient boundary in the es portfolio selection model. J Financ Quant Anal 7(4):1881-1896

Huang Y, Kou G (2014) A kernel entropy manifold learning approach for financial data analysis. Decis Support Syst 64:31-42 
Huang Y, Kou G, Peng Y (2017) Nonlinear manifold learning for early warnings in financial markets. Eur J Oper Res 258(2):692-702

Jiang S, Ong Y-S, Zhang J, Feng L (2014) Consistencies and contradictions of performance metrics in multiobjective optimization. IEEE Trans Cybern 44(12):2391-2404

Kalayci CB, Ertenlice O, Akbay MA (2019) A comprehensive review of deterministic models and applications for mean-variance portfolio optimization. Expert Syst Appl 125:345-368

Konno H, Waki H, Yuuki A (2002) Portfolio optimization under lower partial risk measures. Asia-Pac Financ Mark 9(2):127-140

Kou G (2019) Machine learning methods combined with financial systemic risk. Technol Econ Dev Econ. https://doi.org/ 10.3846/tede.2019.8740

Krink T, Paterlini S (2011) Multiobjective optimization using differential evolution for real-world portfolio optimization. Comput Manag Sci 8(1-2):157-179

Krokhmal P, Palmquist J, Uryasev S (2002) Portfolio optimization with conditional value-at-risk objective and constraints. J Risk 4:43-68

Larsen N, Mausser H, Uryasev S (2002) Algorithms for optimization of value-at-risk. In: Financial Engineering, E-commerce and Supply Chain. Springer, Boston. pp 19-46

Li H, Zhang Q (2009) Multiobjective optimization problems with complicated pareto sets, MOEA/D and NSGA-II. IEEE Trans Evol Comput 13(2):284-302

Liagkouras K, Metaxiotis K (2013) The constrained mean-semivariance portfolio optimization problem with the support of a novel multiobjective evolutionary algorithm. J Softw Eng Appl 6(07):22-29

Liagkouras K, Metaxiotis K (2015) Efficient portfolio construction with the use of multiobjective evolutionary algorithms: best practices and performance metrics. Int J Inf Technol Dec Making 14(03):535-564

Lim C, Sherali HD, Uryasev S (2010) Portfolio optimization by minimizing conditional value-at-risk via nondifferentiable optimization. Comput Optim Appl 46(3):391-415

Liu J-L, Chou C-W, Chen C-M (2010) Optimising mobile base station placement using an enhanced multi-objective genetic algorithm. Int J Bus Intell Data Mining 5(1):19-42

Macedo LL, Godinho P, Alves MJ (2017) Mean-semivariance portfolio optimization with multiobjective evolutionary algorithms and technical analysis rules. Expert Syst Appl 79:33-43

Markowitz H (1959) Portfolio Selection: Efficient Diversification of Investments. Wiley, New York

Markowitz H, Todd P, Xu G, Yamane Y (1993) Computation of mean-semivariance efficient sets by the critical line algorithm. Ann Oper Res 45(1):307-317

Meghwani SS, Thakur M (2017) Multi-criteria algorithms for portfolio optimization under practical constraints. Swarm Evol Comput 37:104-125

Meghwani SS, Thakur M (2018) Multi-objective heuristic algorithms for practical portfolio optimization and rebalancing with transaction cost. Appl Soft Comput 67:865-894

Metaxiotis K, Liagkouras K (2012) Multiobjective evolutionary algorithms for portfolio management: A comprehensive literature review. Expert Syst Appl 39(14):11685-11698

Meucci A (2009) Risk and Asset Allocation. Springer, Berlin

Mezura-Montes E, Coello CAC (2011) Constraint-handling in nature-inspired numerical optimization: past, present and future. Swarm Evol Comput 1(4):173-194

Mishra SK, Panda G, Majhi R (2014) A comparative performance assessment of a set of multiobjective algorithms for constrained portfolio assets selection. Swarm Evol Comput 16:38-51

Mühlenbein H, Schlierkamp-Voosen D (1993) Predictive models for the breeder genetic algorithm i continuous parameter optimization. Evol Comput 1(1):25-49

Nawrocki DN (1991) Optimal algorithms and lower partial moment: ex post results. Appl Econ 23(3):465-470

Nawrocki DN (1999) A brief history of downside risk measures. J Invest 8:9-25

Ponsich A, Jaimes AL, Coello CAC (2013) A survey on multiobjective evolutionary algorithms for the solution of the portfolio optimization problem and other finance and economics applications. IEEE Trans Evol Comput 17(3):321-344

Qin Q, Li L, Cheng S (2014) A novel hybrid algorithm for mean-cvar portfolio selection with real-world constraints. In: Conference in Swarm Intelligence. Springer, Cham. pp 319-327

Rockafellar RT, Uryasev S (2002) Conditional value-at-risk for general loss distributions. J Bank Financ 26(7):1443-1471

Rockafellar RT, Uryasev S, et al (2000) Optimization of conditional value-at-risk. J Risk 2:21-42

Sarykalin S, Serraino G, Uryasev S (2008) Value-at-risk vs. conditional value-at-risk in risk management and optimization. In: State-of-the-Art Decision-Making Tools in the Information-Intensive Age, Tutor Oper Res. INFORMS, Catonsville. pp 270-29

Schott JR (1995) Fault tolerant design using single and multicriteria genetic algorithm optimization. Master's thesis, Department of Aeronautics and Astronautics, Massachussetts Institute of Technology, Cambridge, MA

Schwefel HP (1987) Collective Phenomena in Evolutionary Systems. In: Preprints of the 31st Annual Meeting of the International Society for General System Research, Budapest Vol. 2. pp 1025-1033

Senhaji K, El Moutaouakil K, Ettaouil M (2016) Portfolio selection problem: New multicriteria approach for the mean-semivariance model. In: Logistics Operations Management (GOL), 2016 3rd International Conference On. IEEE Press, Piscataway. pp 1-6

Sing TF, Ong SE (2000) Asset allocation in a downside risk framework. J Real Estate Portfolio Manag 6(3):213-223

Steuer RE, Qi Y, Hirschberger M (2005) Multiple objectives in portfolio selection. J Financ Dec Making 1(1):5-20

Subbu R, Bonissone PP, Eklund N, Bollapragada S, Chalermkraivuth K (2005) Multiobjective financial portfolio design: A hybrid evolutionary approach. In: Evolutionary Computation, 2005. The 2005 IEEE Congress On, vol. 2. IEEE Press, Piscataway. pp 1722-1729

Whitley D (1988) Genitor: A different genetic algorithm. In: Proc. Rocky Mountain Conference on Artificial Intelligence. Colorado Institute for Artificial Intelligence, Denver, Colorado. pp 118-130 
Woodside-Oriakhi M, Lucas C, Beasley JE (2011) Heuristic algorithms for the cardinality constrained efficient frontier. Eur J Oper Res 213(3):538-550

Yamai Y, Yoshiba T, et al (2002) Comparative analyses of expected shortfall and value-at-risk: their estimation error, decomposition, and optimization. Monet Econ Stud 20(1):87-121

Zhang H, Kou G, Peng Y (2019) Soft consensus cost models for group decision making and economic interpretations. Eur J Oper Res 277(3):964-980

Zhang Q, Li H (2007) MOEA/D: A multiobjective evolutionary algorithm based on decomposition. IEEE Trans Evol Comput 11(6):712-731

Zhou A, Jin Y, Zhang Q, Sendhoff B, Tsang E (2006) Combining model-based and genetics-based offspring generation for multi-objective optimization using a convergence criterion. In: Evolutionary Computation, 2006. CEC 2006. IEEE Congress On. IEEE Press, Piscataway. pp 892-899

Zitzler E, Laumanns M, Thiele L (2001) SPEA 2: Improving the strength Pareto evolutionary algorithm. TIK-report 103. ETH Zurich, Zurich

Zitzler E, Thiele L (1999) Multiobjective evolutionary algorithms: a comparative case study and the strength pareto approach. IEEE Trans Evol Comput 3(4):257-271

\section{Publisher's Note}

Springer Nature remains neutral with regard to jurisdictional claims in published maps and institutional affiliations.

Submit your manuscript to a SpringerOpen ${ }^{\circ}$ journal and benefit from:

- Convenient online submission

- Rigorous peer review

- Open access: articles freely available online

- High visibility within the field

- Retaining the copyright to your article

Submit your next manuscript at $\boldsymbol{\triangleright}$ springeropen.com 